\title{
In Situ Strain Tuning in hBN-Encapsulated Graphene Electronic Devices
}

\author{
Lujun Wang, ${ }^{* \dagger, \ddagger \odot}$ Simon Zihlmann, ${ }^{\dagger}$ Andreas Baumgartner, ${ }^{\dagger \dagger}$ Jan Overbeck, ${ }^{\dagger, \ddagger, \S}$ Kenji Watanabe, ${ }^{\|}$

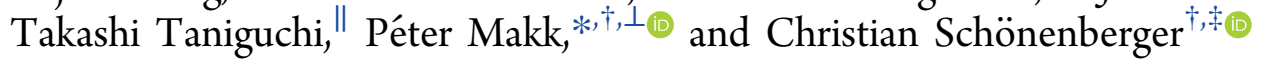

${ }^{\dagger}$ Department of Physics and ${ }^{\ddagger}$ Swiss Nanoscience Institute, University of Basel, Klingelbergstrasse 82, CH-4056 Basel, Switzerland

${ }^{\S}$ Empa, Swiss Federal Laboratories for Materials Science and Technology, Überlandstrasse 129, CH-8600, Dübendorf, Switzerland

"National Institute for Material Science, 1-1 Namiki, Tsukuba 305-0044, Japan

${ }^{\perp}$ Department of Physics, Budapest University of Technology and Economics and Nanoelectronics Momentum Research Group of the Hungarian Academy of Sciences, Budafoki ut 8, 1111 Budapest, Hungary

\section{Supporting Information}

ABSTRACT: Using a simple setup to bend a flexible substrate, we demonstrate deterministic and reproducible in situ strain tuning of graphene electronic devices. Central to this method is the full hBN encapsulation of graphene, which preserves the exceptional quality of pristine graphene for transport experiments. In addition, the on-substrate approach allows one to exploit strain effects in the full range of possible sample geometries and at the same time guarantees that changes in the gate capacitance remain negligible during the deformation process. We use Raman spectroscopy to spatially map the strain magnitude in devices with two different geometries and demonstrate the possibility to engineer a strain gradient, which is relevant for accessing the valley degree of freedom with pseudomagnetic fields. Comparing the transport characteristics of a suspended device with those of an on-substrate device, we demonstrate that our new approach does not suffer from the ambiguities encountered in suspended devices.

KEYWORDS: hBN-encapsulated graphene, strain engineering, $v d W$ heterostructure, Raman spectroscopy, pseudomagnetic field
$\mathrm{T}^{\mathrm{k}}$ he large mechanical strength of two-dimensional (2D) crystals allows one to modify their optical and electronic properties by externally induced strain fields. ${ }^{1}$ Graphene, one of the key examples of $2 \mathrm{D}$ materials, is of particular interest because of its peculiar electronic properties. ${ }^{2}$ A series of intriguing effects were predicted for strained graphene, such as the appearance of a scalar potential, ${ }^{3}$ pseudomagnetic fields, ${ }^{3-5}$ valley filtering, ${ }^{6,7}$ or superconductivity. ${ }^{8}$ Different methods have been introduced to generate strain in graphene. One common approach is based on suspended graphene, where strain is induced by using different microactuators ${ }^{9-12}$ or by simply bending a flexible substrate. ${ }^{13}$ In other approaches, graphene is not suspended and strain can be generated by bending a flexible substrate, ${ }^{14}$ by using highly stressed metallic pads, ${ }^{15}$ or by placing graphene on periodic structures. ${ }^{16-18}$ However, several challenges that need to be overcome simultaneously hampered the progress of these platforms for studying strain effects in transport experiments. First, complex fabrication usually significantly degrades the graphene quality and hinders the observation of the strain effects. In addition, the device is often limited to very basic structures, without the possibility of local gating or multiterminal devices. Second, mechanical deformations often result in changes in the gate capacitance that cannot be easily distinguished from the actual strain effects. The third challenge is that the strain should be in situ tunable and nonhysteretic to disentangle strain effects from other effects.

Here we report a straining method that meets all of the above requirements. Instead of suspending the graphene, we encapsulate the graphene with hexagonal boron-nitride (hBN) and directly strain the van der Waals (vdW) heterostructure on-substrate. This approach preserves the exceptional quality of pristine graphene, which is a significant advantage for transport experiments. We use Raman spectroscopy to demonstrate that our method is versatile and allows one to engineer various strain fields, such as strain gradients, which are important for the generation of pseudomagnetic fields. ${ }^{3} \mathrm{We}$ also show that the edge contacts work reliably and can sustain strain up to $\sim 1 \%$. In the first low-temperature electron transport measurements, we demonstrate that our method solves the problem of an artificial gating effect due to the bending-induced change in the graphene-to-gate distance, which occurs in suspended graphene devices. Therefore, our

Received: April 11, 2019

Revised: May 15, 2019

Published: May 22, 2019 
approach of on-substrate encapsulated graphene offers an ideal platform for studying strain effects in transport experiments.

As shown schematically in Figure $1 \mathrm{a}$, we fabricate hBNencapsulated graphene devices with edge contacts ${ }^{19}$ and an
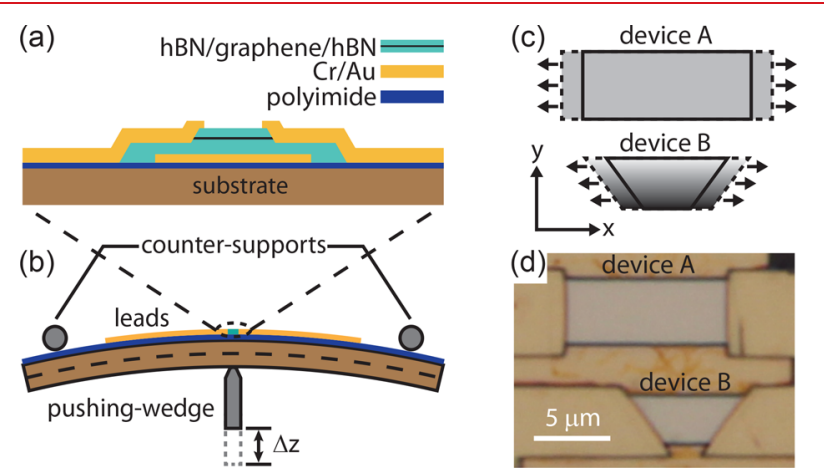

Figure 1. (a) Schematic cross section of our device and (b) of the three-point bending setup. The bending is described by the displacement of the pushing-wedge, $\Delta z$. (c) Illustration of the mechanism for different strain fields. The solid lines represent devices without strain while the dashed lines stand for strained devices. The arrows indicate the elongation of the device along the contacts. The magnitude of strain is shown in grayscale with black corresponding to large strain. (d) Micrograph of two typical devices with different geometries.

electrical bottom gate on a polyimide-coated phosphor bronze substrate. We use exfoliated graphene from natural graphite and usually choose $\sim 20 \mathrm{~nm}$ thick $\mathrm{hBN}$ for the top layer and $\sim 30 \mathrm{~nm}$ thick $\mathrm{hBN}$ for the bottom layer. Bending the substrate in a three-point bending setup, as illustrated in Figure 1b, generates a strain field in the graphene. The deformation of the substrate is determined by the displacement $\Delta z$ of the pushingwedge relative to the mounting position. The hBN encapsulation ensures the high quality of the graphene, and the metallic contacts and the gate allow us to perform transport experiments while tuning the strain in situ. The metallic contacts are essential for generating strain in a vdW heterostructure. In the Supporting Information Figure S3, we present a comparison between devices with and without contacts. The result shows that strain cannot be induced by substrate bending in devices without contacts. On the basis of the assumption that the graphene sheet is pulled uniaxially by the contacts during the bending of the substrate, we designed devices with two different geometries in order to obtain different strain fields. This is illustrated in Figure 1c, where the rectangle (device $A$ ) is expected to result in a homogeneous strain field, while the trapezoid (device B) should exhibit a strain gradient along the $y$-axis, that is, perpendicular to the straining axis. An optical micrograph of two fabricated devices is shown in Figure 1d.

To characterize the strain fields for different displacements $\Delta z$, we perform Raman spectroscopy directly on a three-point bending setup at room temperature. Previous studies reported characteristic redshifts in the Raman peaks of graphene, ${ }^{14,20,21}$ which we now use to quantify the local strain generated in these two devices. Typical Raman spectra of the hBN peak and
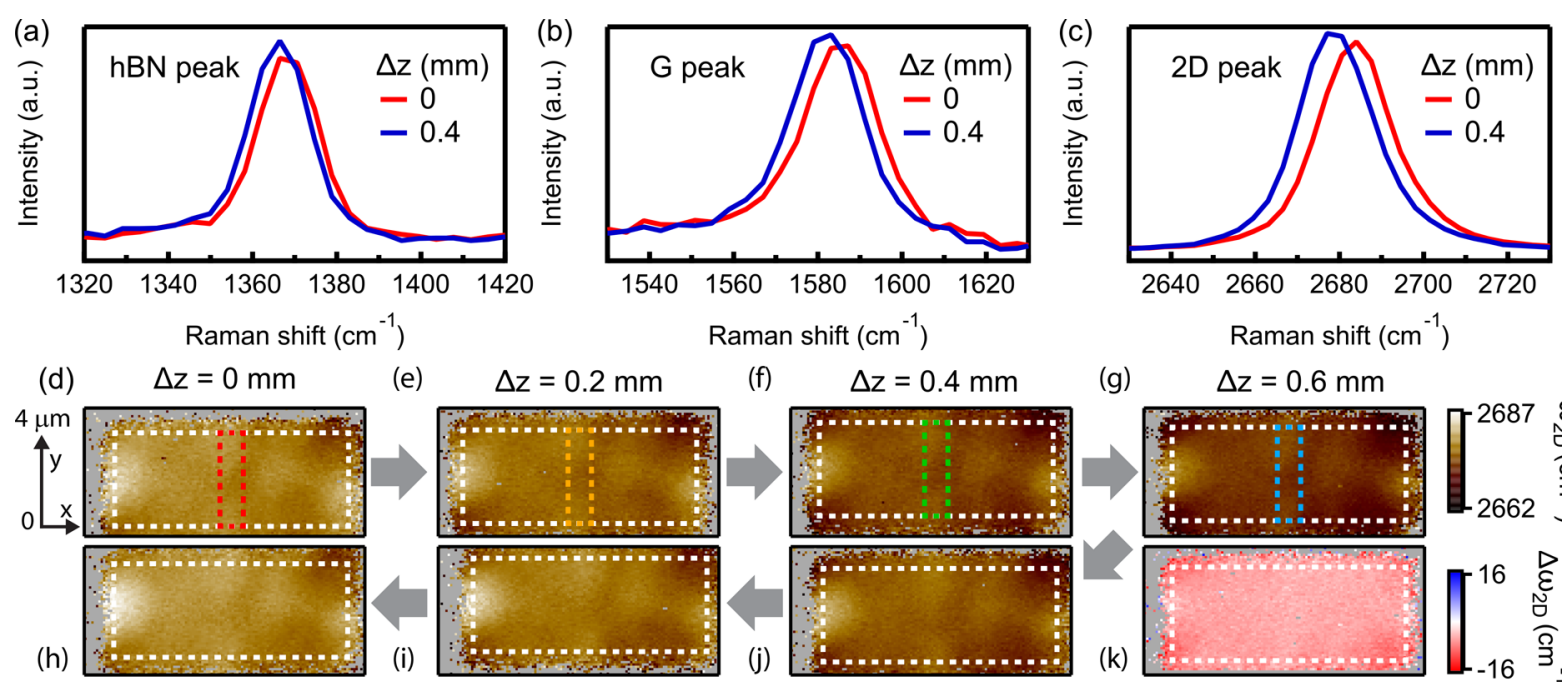

(e) $\Delta z=0.2 \mathrm{~mm}$

(f) $\Delta z=0.4 \mathrm{~mm}$
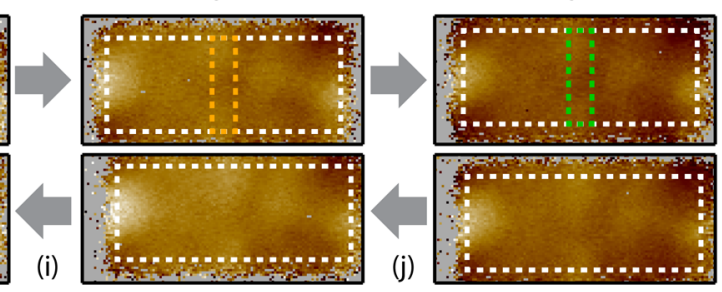

(g)
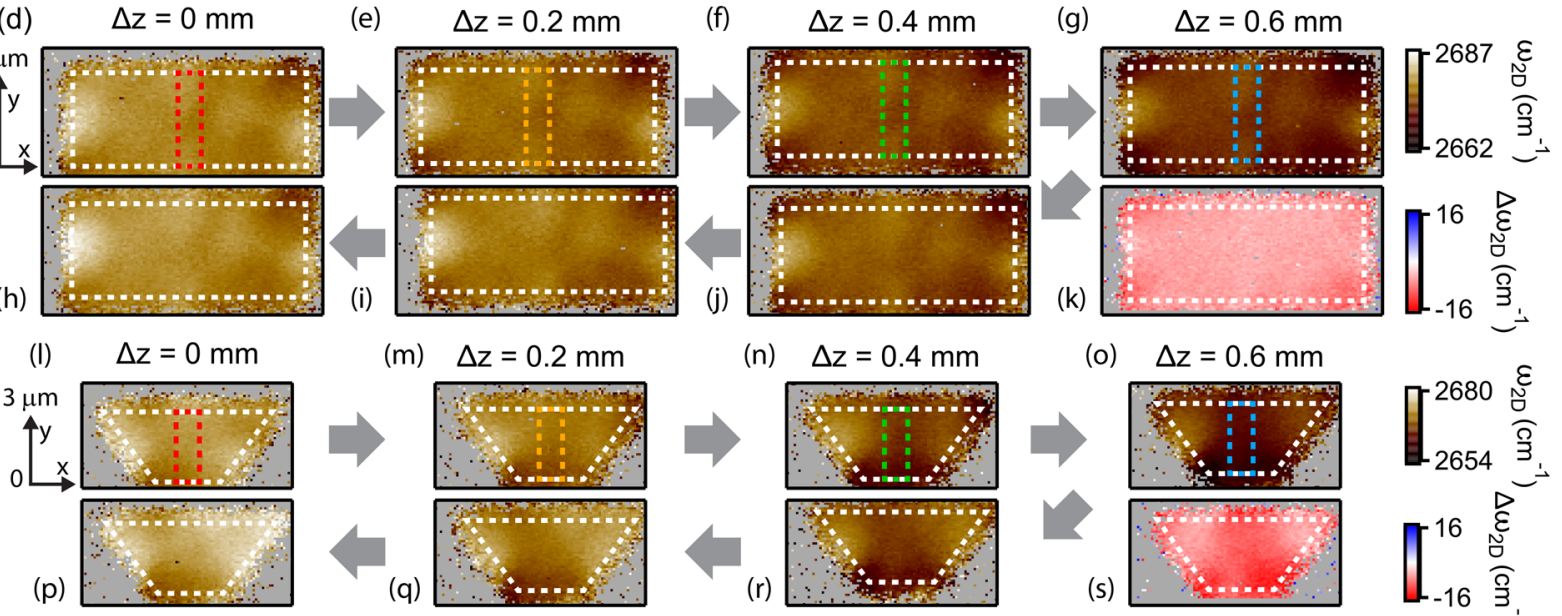

(o) $\Delta z=0.6 \mathrm{~mm}$

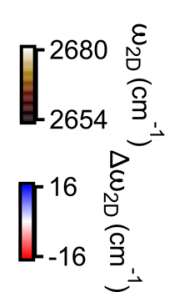

Figure 2. (a-c) Typical Raman spectra of an encapsulated device for two different $\Delta z$ values, zoomed in to the hBN peak, the graphene $\mathrm{G}$ peak and 2D peak, respectively. $(\mathrm{d}-\mathrm{j})$ Spatially resolved Raman maps of $\omega_{2 \mathrm{D}}$ for device A at different $\Delta z$ values. The white dashed lines outline the device boundaries and the colored dashed boxes mark the positions of the profiles shown in Figure 3 . The gray arrows show the sequence of the measurements. (k) Spatially resolved Raman maps of $\Delta \omega_{2 \mathrm{D}}$ for device A, obtained by subtracting map (d) from map (g). (l-r) Spatially resolved Raman maps of $\omega_{2 \mathrm{D}}$ for device B at different $\Delta z$ values. (s) Spatially resolved Raman maps of $\Delta \omega_{2 \mathrm{D}}$ for device B, obtained by subtracting map (l) from map (o). 
(a)

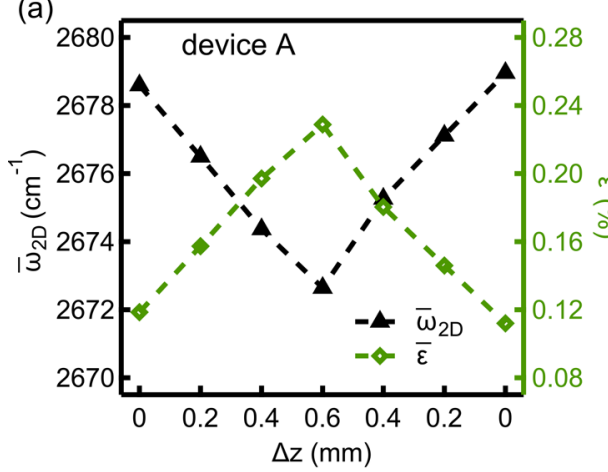

(d)

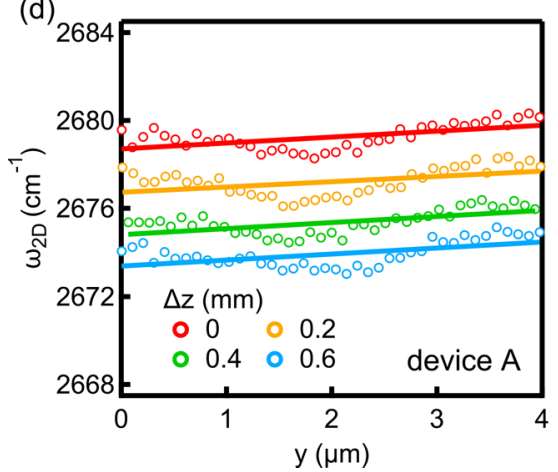

(b)

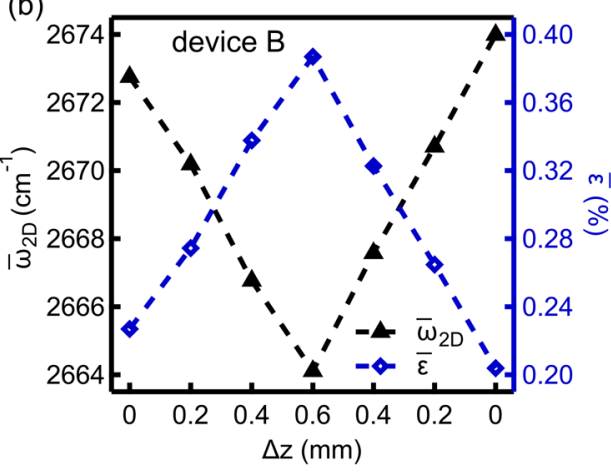

(c)

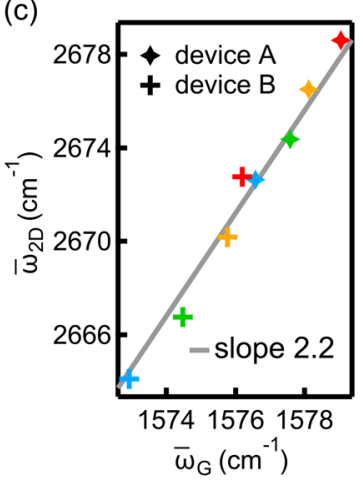

(e)

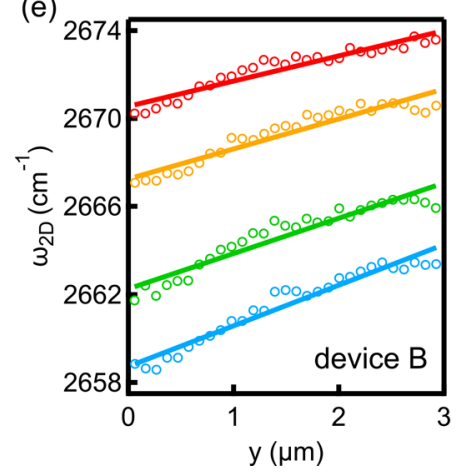

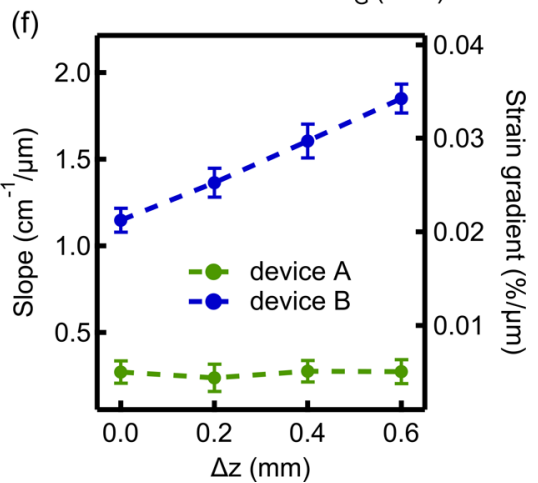

Figure 3. (a,b) $\bar{\omega}_{2 \mathrm{D}}$ and the corresponding strain values plotted as a function of $\Delta z$ for devices A and B, respectively. (c) $\bar{\omega}_{2 \mathrm{D}}$ versus $\bar{\omega}_{\mathrm{G}}$ at different $\Delta z$ for both devices; the gray line has a slope of 2.2. (d,e) Profiles at the center of the maps indicated in Figure 2 for $\Delta z=0 \mathrm{~mm}$ (red), $0.2 \mathrm{~mm}$ (orange), $0.4 \mathrm{~mm}$ (green), and $0.6 \mathrm{~mm}$ (blue). Open circles are data, solid lines are linear fit. (f) Slopes of the profiles plotted as a function of $\Delta z$. The slopes are extracted from linear fitting in $(\mathrm{d}, \mathrm{e})$. The error bars are the fitting errors. The corresponding strain gradient is shown on the right axis.

the graphene $G$ and $2 D$ peaks are shown in Figure $2 a-c$, respectively, for two different $\Delta z$ values. The bending of the substrate results in a redshift of all peaks, with the most prominent effect on the graphene 2D peak. For small strain values, the $2 \mathrm{D}$ peak can be fitted by a single Lorentzian with center frequency $\omega_{2 \mathrm{D}} \cdot{ }^{14}$ In the following, we use spatially resolved Raman spectroscopy to map the strain field based on the redshift of the graphene $2 \mathrm{D}$ peak. The same analysis for the $\mathrm{hBN}$ Raman peak is presented in the Supporting Information Figure S5, which shows that both $\mathrm{hBN}$ and graphene are strained similarly in this method.

We first focus on the investigation of the rectangular device A. In Figure $2 \mathrm{~d}-\mathrm{j}, \omega_{2 \mathrm{D}}$ is plotted as a function of position for device A, for a series of increasing $\Delta z$ from 0 to $0.6 \mathrm{~mm}$ (straining) and then decreasing back to $0 \mathrm{~mm}$ (relaxing), as indicated by the gray arrows. With increasing $\Delta z, \omega_{2 \mathrm{D}}$ shifts to lower values at all positions on the map, consistent with increasing strain everywhere in the graphene sheet. When $\Delta z$ is decreased back to $0 \mathrm{~mm}, \omega_{2 \mathrm{D}}$ reverts back to the initial values. The mean value $\bar{\omega}_{2 \mathrm{D}}$ averaged over the whole device is plotted as a function of $\Delta z$ in Figure 3a, where $\Delta z$ is first increased from 0 to $0.6 \mathrm{~mm}$ then decreased back to $0 \mathrm{~mm}$. The very symmetric V-shape reveals a linear dependence and a good reproducibility of the strain tuning in the graphene device and exhibits no significant hysteresis. This process was repeated several times (up to 8 cycles) and no degradation was observed. The corresponding average strain values $(\bar{\varepsilon})$ are shown on the right axis, which are calculated using $\partial \omega_{2 \mathrm{D}} / \partial \varepsilon=$ $-54 \mathrm{~cm} / \%^{22}$ with $\omega_{2 \mathrm{D}} \approx(2685 \pm 4.4) \mathrm{cm}^{-1}$ for unstrained graphene. We note that the former value is not known very accurate and our choice is among the intermediate reported values. $^{12,14,20-24}$ The latter value is obtained as the average over 10 Raman spectra measured at different positions on 3 different hBN/graphene/hBN stacks before fabrication. The strain values at $\Delta z=0 \mathrm{~mm}$ are not 0 , probably due to the intrinsic strain accumulated in the device after fabrication. We obtain an average strain of up to $0.23 \%$ and a maximum strain near the contacts of $0.3 \%$ for device A at $\Delta z=0.6 \mathrm{~mm}$. Figure $3 \mathrm{c}$ shows the plot of $\bar{\omega}_{2 \mathrm{D}}$ versus $\bar{\omega}_{\mathrm{G}}$ (center frequency of the graphene $\mathrm{G}$ peak) of both devices for different $\Delta z$ values. The data points fall on a line of slope 2.2 , which confirms strain as the origin of the redshift of the Raman peaks. ${ }^{1,25-27}$

We note that there is a small inhomogeneity in the map of $\omega_{2 \mathrm{D}}$ for device $\mathrm{A}$ in Figure $2 \mathrm{~d}$ which does not change significantly with external straining. We analyze the data in the scatter plot of $\omega_{2 \mathrm{D}}$ versus $\omega_{\mathrm{G}}$ in the Supporting Information Figure S4, which shows that the inhomogeneity in the maps of $\omega_{2 \mathrm{D}}$ originates mostly from the strain variation over the large device area. $^{25-28}$ The homogeneity of the externally induced strain field can be seen directly in Figure $2 \mathrm{k}$, which shows a map of the change in the Raman shift, $\Delta \omega_{2 \mathrm{D}}$, between the map at $\Delta z=0.6 \mathrm{~mm}$ (Figure $2 \mathrm{~g}$ ) and the map without external straining (Figure 2d). For this rectangular geometry, the externally induced strain is fairly homogeneous in the bulk with a vanishing strain gradient, which matches quite well the expected strain fields for device A as shown in Figure 1c. The detailed strain pattern for this geometry from finite element method (FEM) simulations is shown in Supporting Information Figure S6.

We now turn to the investigation of strain and strain gradients in the trapezoidal device $\mathrm{B}$. The spatially resolved maps of $\omega_{2 \mathrm{D}}$ for device $\mathrm{B}$ are plotted in Figure 2l-r for the 
same series of $\Delta z$ as above for sample A. Also, this device shows a tunable average strain controlled by $\Delta z$. The device averaged $\bar{\omega}_{2 \mathrm{D}}$ and the corresponding extracted strain values are plotted in Figure $3 \mathrm{~b}$ as a function of $\Delta z$. At identical $\Delta z$ values, the average strain for device $B$ is larger than that for device $A$ due to the smaller size of device $\mathrm{B}$, but shows a similar $\mathrm{V}$ shape, that is, a linear, nonhysteretic dependence on $\Delta z$. These findings can also be seen directly in the Raman maps. We obtain an average strain of up to $0.38 \%$ and maximum values at the lower sample edge of $0.52 \%$ for device B at $\Delta z=0.6 \mathrm{~mm}$. The existence of a strain gradient is visible in Figure $2 \mathrm{~m}-\mathrm{o}$. At the shorter (bottom) edge of the device, $\omega_{2 \mathrm{D}}$ shows a stronger shift than that at the longer (top) edge, which matches the predicted strain pattern for a trapezoidal geometry, as illustrated in Figure 1c (see also the FEM simulations in Supporting Information Figure S6). Figure $2 \mathrm{~s}$ shows the difference between the Raman signals at large bending (Figure 2o) and no bending (Figure 2l).

To demonstrate the deterministic generation of a strain gradient in more detail, we plot $\omega_{2 \mathrm{D}}$ for both devices in Figure $3 \mathrm{~d}$,e as a function of the position along the $y$-axis in the center of the device area, averaged over $1 \mu \mathrm{m}$ in the $x$-direction, as indicated by the colored dashed boxes in Figure $2 \mathrm{~d}-\mathrm{g}, 1-\mathrm{o}$. For both devices, we find a clear increase in the average strain (overall shift of the curves) and an essentially unchanged background variation with increasing $\Delta z$. In addition, for the trapezoidal geometry (device B) we find a clear linear increase in the strain when moving from the longer to the shorter sample edge.

We now take the average slope of these curves as an estimate of the large scale (nonmicroscopic) strain gradient along the $y$ axis. For this purpose, we plot in Figure $3 \mathrm{f}$ the slopes of linear fits to the data in Figure $3 \mathrm{~d}$ (device A) and Figure $3 \mathrm{e}$ (device B) as a function of $\Delta z$ with the right axis showing the corresponding extracted strain gradient. The small nonzero slope for device A stems from the small intrinsic strain variation over the large device area discussed above and stays constant for increasing $\Delta z$. In contrast, for device B we find a linear dependence of the average slope on the $\Delta z$, demonstrating that strain gradients can be generated by modifying the device geometry. The pseudomagnetic field, $B_{\mathrm{ps}}$, depends on the strain gradients, $B_{\mathrm{ps}}=\frac{\hbar \beta}{e a}\left[-2 \frac{\partial \varepsilon_{x y}}{\partial x}-\frac{\partial\left(\varepsilon_{x x}-\varepsilon_{y y}\right)}{\partial y}\right], 3,4$ with $\beta \approx 3.37$ as the Grüneisen parameter ${ }^{29}$ and $a=1.42 \AA$ as the interatomic distance. To estimate $B_{\mathrm{ps}}$, we use $B_{\mathrm{ps}} \approx \frac{\hbar \beta}{e a} \frac{\partial \varepsilon_{x x}}{\partial y}$ and obtain values on the order of $10 \mathrm{mT}$ in the bulk of device B at $\Delta z=$ $0.6 \mathrm{~mm}$. We note that much larger strain gradients occur near the corners of the device, which might result in local pseudomagnetic fields larger than $100 \mathrm{mT}$. We also point out that larger average strain values can be achieved in this setup (see Supporting Information Figure S7) but with an increased probability of device failure, which happens at a strain on the order of $1 \%$.

One of the major advantages of our technique is that the edge contacts not only act as clamps for the mechanical deformation but also allow for simultaneous transport experiments. Here, we report the first low-temperature $(4 \mathrm{~K})$ transport experiments with in situ strain tuning of a device fabricated in the described fashion and compare the results to similar measurements on a device where the encapsulated graphene is suspended, using the LOR-based suspension

technique. ${ }^{30,31}$ The two experiments are shown schematically in Figure $4 a, b$. For both devices the two-terminal differential

(a)

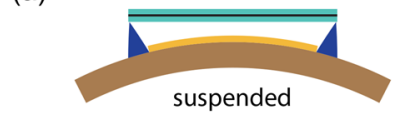

(c)

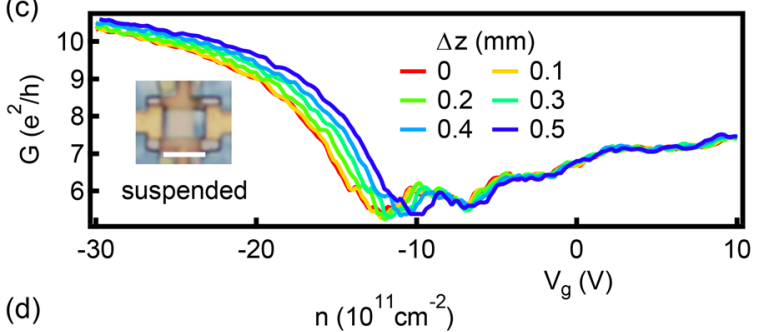

(d)

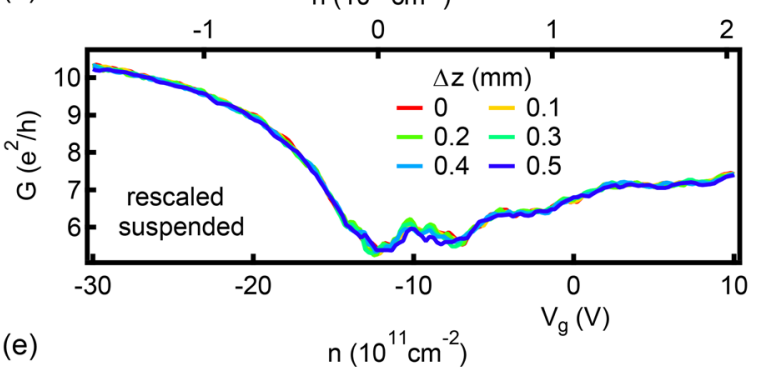

(e)

(b)
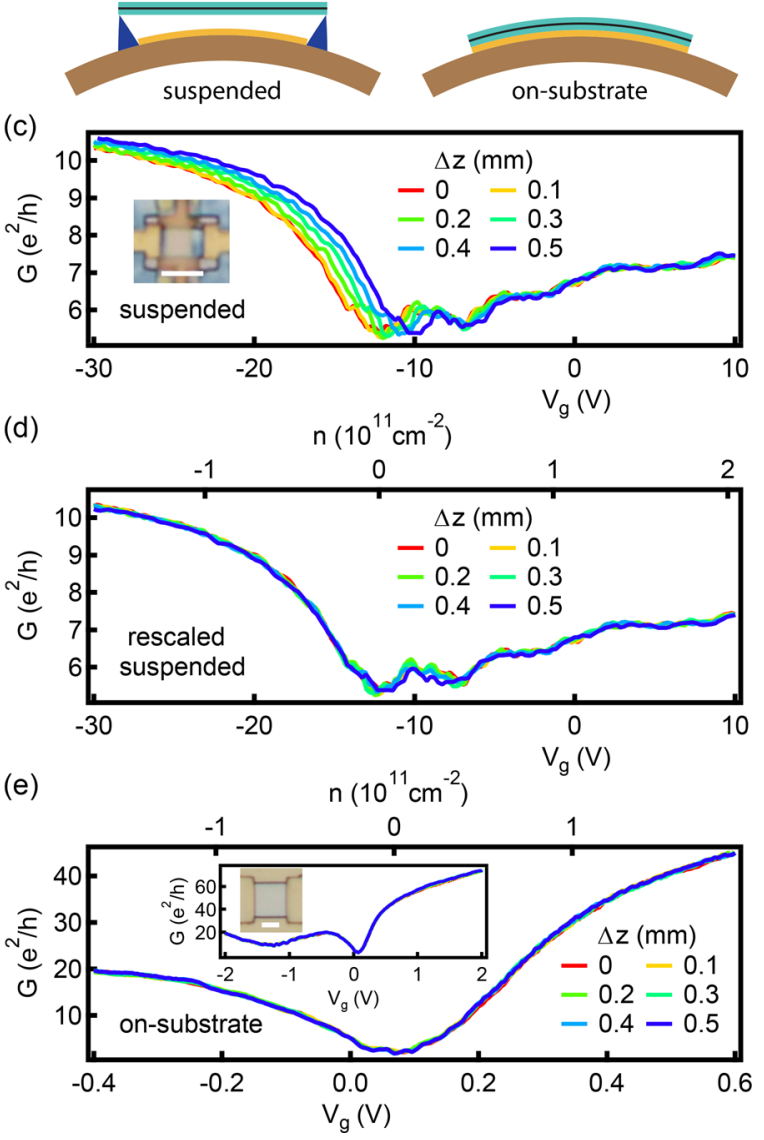

Figure 4. (a,b) Illustration of substrate bending for suspended and on-substrate devices, respectively. The graphene-to-gate distance changes with substrate bending for suspended devices while it remains unchanged for on-substrate devices. (c) Two-terminal differential conductance, $G$, plotted as a function of gate voltage, $V_{\mathrm{g}}$, for a suspended device for different $\Delta z$ values. The inset is the micrograph of the measured device. (d) The same data as in (c) with the curves rescaled in $V_{\mathrm{g}}$ with respect to $V_{\mathrm{g}}=0 \mathrm{~V}$ by matching the CNP of each curve with that of the curve at $\Delta z=0 \mathrm{~mm}$. The corresponding carrier density is shown on the top axis. (e) $G$ of an on-substrate device for a similar charge carrier density range for different $\Delta z$ values. The inset shows $G$ on a larger $V_{\mathrm{g}}$ range. The micrograph is the measured device. The scale bars correspond to $2 \mu \mathrm{m}$.

conductance, $G$, is measured as a function of the gate voltage, $V_{\mathrm{g}}$, for different $\Delta z$ values using standard low-frequency lock-in techniques (see Figure $4 \mathrm{c}, \mathrm{e}$ ).

There are significant differences between suspended and onsubstrate devices for bending experiments. For the suspended device, one can immediately find a systematic change of the curves in gate voltage with increasing $\Delta z$ (see Figure 4c), whereas such an obvious effect is absent for the on-substrate device (see Figure 4e). The effects found in the suspended device can be fully accounted for by the change in the graphene-to-gate distance when bending the substrate, as depicted in Figure 4a. This is illustrated in Figure 4d, where we plot the data of Figure $4 \mathrm{c}$ rescaled linearly in gate voltage for each curve with $V_{\mathrm{g}}=0 \mathrm{~V}$ as a fixed point. This can be understood in a simple capacitor model in which the charge induced in the graphene is given by $Q=C V_{\mathrm{g}}$ with $C$ as the 
effective capacitance between the graphene layer and the gate. If the capacitance is changed by a factor $\alpha$ to $\alpha C$ due to the substrate bending, the same charge $Q$ is induced at $V_{\mathrm{g}} / \alpha$, which is equivalent to a rescaling in the gate voltage. This scaling factor is extracted for each curve by matching the CNP to that of the curve at $\Delta z=0 \mathrm{~mm}$ and it is linear in $\Delta z$. After rescaling, all data points fall onto the same curve, see Figure $4 \mathrm{~d}$. This demonstrates that the bending-induced gating effect is dominant for the suspended graphene device, which makes it very difficult to study effects due to actual strain.

This effect is absent in the on-substrate devices optimized for strain tuning. For comparison, we performed the same type of measurements also on an on-substrate device with the results shown in Figure 4e. Because the gate voltage lever arm in this device is much larger than that for the suspended device due to the shorter graphene-to-gate distance, we apply smaller gate voltages to obtain a similar carrier density range as that in the data of the suspended device (see top axes of Figure 4d,e). A electron mobility of $\sim 100000 \mathrm{~cm}^{2} \mathrm{~V}^{-1} \mathrm{~s}^{-1}$ is extracted from a linear fit around the CNP, suggesting a high graphene quality in our device. On this gate voltage scale and also on a much larger scale (see inset of Figure 4e), all curves with different $\Delta z$ values are virtually identical, which demonstrates that there are no bending-induced changes in the carrier density or in the contact resistance in this experimental configuration. The additional conductance minimum at $V_{\mathrm{g}} \approx 1.3 \mathrm{~V}$ comes from a double moiré superlattice effect in encapsulated graphene. ${ }^{32}$ We point out that on this scale of graphene straining we could not detect significant changes in the conductance.

In conclusion, we have successfully generated tunable and reversible strain fields in encapsulated graphene devices. Because these devices are fabricated on-substrate, that is, not suspended, it allows us to design a large variety of device geometries. As an example, we use spatially resolved Raman imaging to demonstrate that the edge contact clamping and rectangular geometry result in a fairly homogeneous straining of the graphene. In a second step, we use this design freedom to generate a strain gradient in a trapezoidal geometry. In first transport experiments, we then demonstrate another major advantage of on-substrate encapsulated devices, namely, that the bending-induced gate capacitance change can be avoided, which is crucial for studying strain effects in transport experiments. This approach is not limited to graphene but also is suitable for studying strain effects in other $2 \mathrm{D}$ materials and complex vdW heterostructures, for example, in $\mathrm{MoS}_{2}{ }^{33-35}$ Because our method is simple and intuitive, nonetheless allowing complex device structures, we expect that it will pave the way toward deterministic strain engineering and new approaches to valleytronics.

\section{ASSOCIATED CONTENT}

\section{S Supporting Information}

The Supporting Information is available free of charge on the ACS Publications website at DOI: 10.1021/acs.nanolett.9b01491.

Details of the fabrication and the measurement setups, comparison between devices with and without contacts, discussions of the correlation between $\omega_{2 \mathrm{D}}$ and $\omega_{\mathrm{G}}$, analysis of the hBN Raman maps, FEM simulations, contact failure, and discussions of the suspended encapsulated device (PDF)

\section{AUTHOR INFORMATION}

\section{Corresponding Authors}

*E-mail: lujun.wang@unibas.ch.

*E-mail: peter.makk@unibas.ch.

ORCID

Lujun Wang: 0000-0002-5447-0484

Péter Makk: 0000-0001-7637-4672

Christian Schönenberger: 0000-0002-5652-460X

\section{Author Contributions}

L.W. fabricated the devices, performed the measurements, and did the data analysis. S.Z. initiated the idea of hBNencapsulation. A.B. did the FEM simulations. P.M and J.O. helped to develop the straining method. P.M., S.Z. and J.O. supported the device fabrication. A.B., P.M., and S.Z. helped with the data analysis. K.W. and T.T. provided high-quality hBN. C.S. initiated and supervised the project. L.W. and A.B. wrote the paper. All authors discussed the results and worked on the manuscript.

\section{Notes}

The authors declare no competing financial interest.

All data in this publication are available in numerical form at DOI:https://doi.org/10.5281/zenodo.2636531.

\section{ACKNOWLEDGMENTS}

This work has received funding from the Swiss Nanoscience Institute (SNI), the ERC project TopSupra (787414), the European Union Horizon 2020 research and innovation programme under Grant Agreement 785219 (Graphene Flagship), the Swiss National Science Foundation, the Swiss NCCR QSIT, Topograph, ISpinText FlagERA network and from the OTKA FK-123894 grants. P.M. acknowledges support from the Bolyai Fellowship, the Marie Curie Grant, and the National Research, Development, and Innovation Fund of Hungary within the Quantum Technology National Excellence Program (Project No. 2017-1.2.1-NKP-201700001). Growth of hexagonal boron nitride crystals was supported by the Elemental Strategy Initiative conducted by the MEXT, Japan and the CREST (JPMJCR15F3), JST. The authors thank Oliver Braun, David Indolese and Peter Rickhaus for fruitful discussions, and Sascha Martin and his team for their technical support.

\section{REFERENCES}

(1) Roldán, R.; Castellanos-Gomez, A.; Cappelluti, E.; Guinea, F. Strain engineering in semiconducting two-dimensional crystals. J. Phys.: Condens. Matter 2015, 27, 313201.

(2) Castro Neto, A. H.; Guinea, F.; Peres, N. M. R.; Novoselov, K. S.; Geim, A. K. The electronic properties of graphene. Rev. Mod. Phys. 2009, 81, 109-162.

(3) Guinea, F.; Geim, A. K.; Katsnelson, M. I.; Novoselov, K. S. Generating quantizing pseudomagnetic fields by bending graphene ribbons. Phys. Rev. B: Condens. Matter Mater. Phys. 2010, 81, 035408.

(4) Guinea, F.; Katsnelson, M. I.; Geim, A. K. Energy gaps and a zero-field quantum Hall effect in graphene by strain engineering. Nat. Phys. 2010, 6, 30.

(5) Zhu, S.; Stroscio, J. A.; Li, T. Programmable Extreme Pseudomagnetic Fields in Graphene by a Uniaxial Stretch. Phys. Rev. Lett. 2015, 115, 245501.

(6) Milovanović, S. P.; Peeters, F. M. Strain controlled valley filtering in multi-terminal graphene structures. Appl. Phys. Lett. 2016, 109, 203108. 
(7) Yesilyurt, C.; Ghee Tan, S.; Liang, G.; Jalil, M. B. A. Perfect valley filter in strained graphene with single barrier region. AIP Adv. 2016, 6, 056303.

(8) Uchoa, B.; Barlas, Y. Superconducting States in Pseudo-LandauLevels of Strained Graphene. Phys. Rev. Lett. 2013, 111, 046604.

(9) Pérez Garza, H. H.; Kievit, E. W.; Schneider, G. F.; Staufer, U. Controlled, Reversible, and Nondestructive Generation of Uniaxial Extreme Strains (>10in Graphene. Nano Lett. 2014, 14, 4107-4113.

(10) Downs, C. S. C.; Usher, A.; Martin, J. Towards observation of pseudo-magnetic fields in suspended graphene devices. J. Appl. Phys. 2016, 119, 194305.

(11) Goldsche, M.; Sonntag, J.; Khodkov, T.; Verbiest, G. J.; Reichardt, S.; Neumann, C.; Ouaj, T.; von den Driesch, N.; Buca, D.; Stampfer, C. Tailoring Mechanically Tunable Strain Fields in Graphene. Nano Lett. 2018, 18, 1707-1713.

(12) Colangelo, F.; Pitanti, A.; Mišeikis, V.; Coletti, C.; Pingue, P.; Pisignano, D.; Beltram, F.; Tredicucci, A.; Roddaro, S. Controlling local deformation in graphene using micrometric polymeric actuators. $2 D$ Mater. 2018, 5, 045032.

(13) Guan, F.; Du, X. Random Gauge Field Scattering in Monolayer Graphene. Nano Lett. 2017, 17, 7009-7014.

(14) Mohiuddin, T. M. G.; Lombardo, A.; Nair, R. R.; Bonetti, A.; Savini, G.; Jalil, R.; Bonini, N.; Basko, D. M.; Galiotis, C.; Marzari, N.; Novoselov, K. S.; Geim, A. K.; Ferrari, A. C. Uniaxial strain in graphene by Raman spectroscopy: $G$ peak splitting, Grüneisen parameters, and sample orientation. Phys. Rev. B: Condens. Matter Mater. Phys. 2009, 79, 205433.

(15) Shioya, H.; Russo, S.; Yamamoto, M.; Craciun, M. F.; Tarucha, S. Electron States of Uniaxially Strained Graphene. Nano Lett. 2015, $15,7943-7948$.

(16) Jiang, Y.; Mao, J.; Duan, J.; Lai, X.; Watanabe, K.; Taniguchi, T.; Andrei, E. Y. Visualizing Strain-Induced Pseudomagnetic Fields in Graphene through an hBN Magnifying Glass. Nano Lett. 2017, 17, 2839-2843.

(17) Liu, Y.; Rodrigues, J. N. B.; Luo, Y. Z.; Li, L.; Carvalho, A.; Yang, M.; Laksono, E.; Lu, J.; Bao, Y.; Xu, H.; Tan, S. J. R.; Qiu, Z.; Sow, C. H.; Feng, Y. P.; Neto, A. H. C.; Adam, S.; Lu, J.; Loh, K. P. Tailoring sample-wide pseudo-magnetic fields on a graphene-black phosphorus heterostructure. Nat. Nanotechnol. 2018, 13, 828-834.

(18) Zhang, Y.; Heiranian, M.; Janicek, B.; Budrikis, Z.; Zapperi, S.; Huang, P. Y.; Johnson, H. T.; Aluru, N. R.; Lyding, J. W.; Mason, N. Strain Modulation of Graphene by Nanoscale Substrate Curvatures: A Molecular View. Nano Lett. 2018, 18, 2098-2104.

(19) Wang, L.; Meric, I.; Huang, P. Y.; Gao, Q.; Gao, Y.; Tran, H.; Taniguchi, T.; Watanabe, K.; Campos, L. M.; Muller, D. A.; Guo, J.; Kim, P.; Hone, J.; Shepard, K. L.; Dean, C. R. One-Dimensional Electrical Contact to a Two-Dimensional Material. Science 2013, 342, 614-617.

(20) Yoon, D.; Son, Y.-W.; Cheong, H. Strain-Dependent Splitting of the Double-Resonance Raman Scattering Band in Graphene. Phys. Rev. Lett. 2011, 106, 155502.

(21) Frank, O.; Mohr, M.; Maultzsch, J.; Thomsen, C.; Riaz, I.; Jalil, R.; Novoselov, K. S.; Tsoukleri, G.; Parthenios, J.; Papagelis, K.; Kavan, L.; Galiotis, C. Raman 2D-Band Splitting in Graphene: Theory and Experiment. ACS Nano 2011, 5, 2231-2239.

(22) Mohr, M.; Papagelis, K.; Maultzsch, J.; Thomsen, C. Twodimensional electronic and vibrational band structure of uniaxially strained graphene from ab initio calculations. Phys. Rev. B: Condens. Matter Mater. Phys. 2009, 80, 205410.

(23) Huang, M.; Yan, H.; Heinz, T. F.; Hone, J. Probing StrainInduced Electronic Structure Change in Graphene by Raman Spectroscopy. Nano Lett. 2010, 10, 4074-4079.

(24) Polyzos, I.; Bianchi, M.; Rizzi, L.; Koukaras, E. N.; Parthenios, J.; Papagelis, K.; Sordan, R.; Galiotis, C. Suspended monolayer graphene under true uniaxial deformation. Nanoscale 2015, 7, 1303313042.

(25) Lee, J. E.; Ahn, G.; Shim, J.; Lee, Y. S.; Ryu, S. Optical separation of mechanical strain from charge doping in graphene. Nat. Commun. 2012, 3, 1024.
(26) Neumann, C.; Reichardt, S.; Venezuela, P.; Drögeler, M.; Banszerus, L.; Schmitz, M.; Watanabe, K.; Taniguchi, T.; Mauri, F.; Beschoten, B.; Rotkin, S. V.; Stampfer, C. Raman spectroscopy as probe of nanometre-scale strain variations in graphene. Nat. Commun. 2015, 6, 8429.

(27) Mueller, N. S.; Heeg, S.; Alvarez, M. P.; Kusch, P.; Wasserroth, S.; Clark, N.; Schedin, F.; Parthenios, J.; Papagelis, K.; Galiotis, C.; Kalbáč, M.; Vijayaraghavan, A.; Huebner, U.; Gorbachev, R.; Frank, O.; Reich, S. Evaluating arbitrary strain configurations and doping in graphene with Raman spectroscopy. 2D Mater. 2018, 5, 015016.

(28) Froehlicher, G.; Berciaud, S. Raman spectroscopy of electrochemically gated graphene transistors: Geometrical capacitance, electron-phonon, electron-electron, and electron-defect scattering. Phys. Rev. B: Condens. Matter Mater. Phys. 2015, 91, 205413.

(29) Pereira, V. M.; Castro Neto, A. H.; Peres, N. M. R. Tightbinding approach to uniaxial strain in graphene. Phys. Rev. B: Condens. Matter Mater. Phys. 2009, 80, 045401.

(30) Tombros, N.; Veligura, A.; Junesch, J.; Jasper van den Berg, J.; Zomer, P. J.; Wojtaszek, M.; Vera Marun, I. J.; Jonkman, H. T.; van Wees, B. J. Large yield production of high mobility freely suspended graphene electronic devices on a polydimethylglutarimide based organic polymer. J. Appl. Phys. 2011, 109, 093702.

(31) Maurand, R.; Rickhaus, P.; Makk, P.; Hess, S.; Tóvári, E.; Handschin, C.; Weiss, M.; Schönenberger, C. Fabrication of ballistic suspended graphene with local-gating. Carbon 2014, 79, 486-492.

(32) Wang, L.; Zihlmann, S.; Liu, M.-H.; Makk, P.; Watanabe, K.; Taniguchi, T.; Baumgartner, A.; Schönenberger, C. New Generation of Moiré Superlattices in Doubly Aligned hBN/Graphene/hBN Heterostructures. Nano Lett. 2019, 19, 2371-2376.

(33) Conley, H. J.; Wang, B.; Ziegler, J. I.; Haglund, R. F.; Pantelides, S. T.; Bolotin, K. I. Bandgap Engineering of Strained Monolayer and Bilayer MoS2. Nano Lett. 2013, 13, 3626-3630.

(34) He, K.; Poole, C.; Mak, K. F.; Shan, J. Experimental Demonstration of Continuous Electronic Structure Tuning via Strain in Atomically Thin MoS2. Nano Lett. 2013, 13, 2931-2936.

(35) Zhu, C. R.; Wang, G.; Liu, B. L.; Marie, X.; Qiao, X. F.; Zhang, X.; Wu, X. X.; Fan, H.; Tan, P. H.; Amand, T.; Urbaszek, B. Strain tuning of optical emission energy and polarization in monolayer and bilayer $\mathrm{MoS}_{2}$. Phys. Rev. B: Condens. Matter Mater. Phys. 2013, 88, 121301. 